Western University

Scholarship@Western

Oncology Publications

Oncology Department

$11-15-2007$

\title{
Adaptive radiotherapy planning on decreasing gross tumor volumes as seen on megavoltage computed tomography images.
}

Curtis Woodford

Slav Yartsev

University of Western Ontario, vyartse@uwo.ca

R. Dar

University of Western Ontario, rashid.dar@lhsc.on.ca

Glenn Bauman

University of Western Ontario, glenn.bauman@lhsc.on.ca

Jacob Van Dyk

The University of Western Ontario, vandyk@uwo.ca

Follow this and additional works at: https://ir.lib.uwo.ca/oncpub

Part of the Medical Biophysics Commons, and the Oncology Commons

Citation of this paper:

Woodford, Curtis; Yartsev, Slav; Dar, R.; Bauman, Glenn; and Van Dyk, Jacob, "Adaptive radiotherapy planning on decreasing gross tumor volumes as seen on megavoltage computed tomography images." (2007). Oncology Publications. 135.

https://ir.lib.uwo.ca/oncpub/135 


\title{
PHYSICS CONTRIBUTION
}

doi:10.1016/j.jjrobp.2007.07.2369

\section{ADAPTIVE RADIOTHERAPY PLANNING ON DECREASING GROSS TUMOR VOLUMES AS SEEN ON MEGAVOLTAGE COMPUTED TOMOGRAPHY IMAGES}

\author{
Curtis Woodford, ${ }^{*}$ Slav Yartsev, Ph.D., ${ }^{*}$ A. Rashid Dar, M.D. ${ }^{* \dagger}$ Glenn Bauman, M.D., ${ }^{\dagger}$ \\ AND JAKE VAN DYK, M.SC. ${ }^{\dagger \dagger}$ \\ * London Regional Cancer Program, London Health Sciences Centre, London, Ontario, Canada; and \\ ${ }^{\dagger}$ The University of Western Ontario, London, Ontario, Canada
}

\begin{abstract}
Purpose: To evaluate gross tumor volume (GTV) changes for patients with non-small-cell lung cancer by using daily megavoltage (MV) computed tomography (CT) studies acquired before each treatment fraction on helical tomotherapy and to relate the potential benefit of adaptive image-guided radiotherapy to changes in GTV.

Methods and Materials: Seventeen patients were prescribed 30 fractions of radiotherapy on helical tomotherapy for non-small-cell lung cancer at London Regional Cancer Program from Dec 2005 to March 2007. The GTV was contoured on the daily MVCT studies of each patient. Adapted plans were created using merged MVCT-kilovoltage CT image sets to investigate the advantages of replanning for patients with differing GTV regression characteristics.

Results: Average GTV change observed over 30 fractions was $-38 \%$, ranging from -12 to $-87 \%$. No significant correlation was observed between GTV change and patient's physical or tumor features. Patterns of GTV changes in the $\mathbf{1 7}$ patients could be divided broadly into three groups with distinctive potential for benefit from adaptive planning.

Conclusions: Changes in GTV are difficult to predict quantitatively based on patient or tumor characteristics. If changes occur, there are points in time during the treatment course when it may be appropriate to adapt the plan to improve sparing of normal tissues. If GTV decreases by greater than $30 \%$ at any point in the first 20 fractions of treatment, adaptive planning is appropriate to further improve the therapeutic ratio. 다일 2007 Elsevier Inc.
\end{abstract}

Helical tomotherapy, Lung cancer, Plan reoptimization, Adaptive planning.

\section{INTRODUCTION}

The megavoltage (MV) computed tomography (CT) acquisition capability of helical tomotherapy has proved very effective for pretreatment patient positioning to decrease setup errors $(1,2)$. The MVCT image sets depict the patient's anatomy with image contrast and resolution that is slightly inferior to a kilovoltage $\mathrm{CT}(\mathrm{kVCT})$ study, but has enough soft-tissue contrast to reliably contour organs or lesions in many areas of the body $(3,4)$. In particular, peripheral-lung tumors can be easily detected and delineated on MVCT image sets. However, significant uncertainty in target delineation may be associated with tumors partially abutting or primarily contained in the mediastinum $(5,6)$. If treatment for patients with non-small-cell lung cancer (NSCLC) on helical tomotherapy involves regular MVCT imaging, the gross tumor volume (GTV) response to radiotherapy can potentially be assessed on a daily basis.
Tumor control predictions based on pretreatment measurements are well studied, and most investigators used the criterion of tumor size measured by largest tumor dimension, bidimensional product, or tumor volume for a local control predictor. Usefulness of tumor size/volume as an overall prognostic factor for survival is still under discussion (79). Correlation of pretreatment tumor size with local control suggests that such tumor characteristics as size could possibly be used to anticipate the amount of GTV regression during a course of treatment. Generally, NSCLC tumor response to radiotherapy is believed to be a slow process because tumors reach their maximum response (minimum volume) an average of 11 months after radiotherapy completion (9). Kupelian et al. (10) and Siker et al. (11) used measurements of tumor volume on serial MVCT image sets generated by helical tomotherapy to document interfractional radiation responses during a shorter period, whereas Ramsey et al. (12) focused on adaptive dosimetric planning as tumor
Reprint requests to: Slav Yartsev, Ph.D., London Regional Cancer Program, London Health Sciences Centre, 790 Commissioners Rd East, London, Ontario, Canada N6A 4L6. Tel: (+1) 519-6858605; Fax: (+1) 519-685-8658; E-mail: slav.yartsev@lhsc.on.ca
This study was supported by the Ontario Institute for Cancer Research through funding provided by the government of Ontario. Conflict of interest: none.

Received May 10, 2007, and in revised form July 26, 2007. Accepted for publication July 27, 2007. 
volume changes. Others used multiple kVCT scans to evaluate tumor volume changes during radiotherapy (13-16) or portal images to monitor tumor position, size, and movement (17). All these investigators observed tumor volume shrinkage to varying degrees during the course of fractionated treatment, suggesting tumor volume changes during shorter periods may be clinically relevant. The aim of this report is to evaluate GTV changes in 17 patients treated for NSCLC on helical tomotherapy, characterize GTV variation, and model the potential benefit of adaptive planning during a course of fractionated radiotherapy.

\section{METHODS AND MATERIALS}

Seventeen patients were treated for NSCLC on the Hi-Art helical tomotherapy (TomoTherapy, Inc., Madison, WI) unit at the London Regional Cancer Program, Ontario, Canada, from December 2005 to March 2007. All patients received cisplatin and vinorelbine as neoadjuvant chemotherapy, finishing treatment 4-6 weeks before the start of radiotherapy. Chemotherapy is not expected to affect relative GTV changes from radiotherapy in patients because all patients were treated using the same regimen. The CT simulation and tomotherapy treatment planning (Hi-ART, version 2.2.2) occurred approximately 3 weeks before starting treatment, with delivery quality assurance performed to ensure proper dose distributions and absolute dose delivery. One initial mock treatment that included an MVCT study was performed for each patient, after which they began treatment with daily MVCT acquisitions for setup verification. A prescription dose of 60-64 Gy in 2 Gy/fraction was used for patients in this study, all of whom had locally advanced (Stage $\geq$ IIIA) disease. Elective nodal radiation was included for some patients, with doses of 50 or 60 Gy delivered to adjacent radiographically uninvolved nodal regions. The MVCT scans on the Hi-Art system were acquired with photon beam energy of $3.5 \mathrm{MV}$, field of view of $40 \mathrm{~cm}$, fan beam width of $5 \mathrm{~mm}$ at the isocenter, and pitch factor of 2.4 for coarse (6-mm) slices (3). The reconstruction matrix was $512 \times 512$ in the axial plane, yielding a $0.78 \times 0.78 \times 6-\mathrm{mm}^{3}$ voxel size. For this patient population, Planned Adaptive (TomoTherapy, Inc.) software was not available at the time of treatment. For this reason, an additional kVCT study was performed for 6 patients to construct an adapted plan at the request of the treating radiation oncologist for patients with local anatomy changes deemed clinically significant on serial MVCTs during the actual treatment course.

For this report, GTV volume changes were calculated retrospectively based on the serial MVCTs obtained daily during treatment. For calculation of GTV changes during the course of treatment, GTV was contoured after transferring the daily MVCT studies for each patient from the helical tomotherapy unit to a treatment planning system (either Pinnacle ${ }^{3}$ version 8.0d; Philips, Fitchburg, WI, or Oncentra Treatment Planning, version 1.3.1.13; Nucletron, Veenendaal, The Netherlands). The MVCT images were contoured with a window/level setting of 1600/-300 for tumor bounded by parenchyma and 400/800 for tumor abutting the mediastinum. The kVCT studies for each patient were available to provide guidance to the investigator (C.W.) who did the MVCT contouring. Involved nodes were excluded from the GTV; only primary tumor volume was measured for the purpose of calculating response in this report.

The MVCT images generated by using helical tomotherapy can also be used to create adapted plans when significant changes in the patient's GTV are observed as treatment progresses. In our study, Planned Adaptive software was used to create merged images for 3 patients in which the $40-\mathrm{cm}$ field of view of the pretreatment MVCT replaced the corresponding section of the full planning $\mathrm{kVCT}$. The registration values used to position patients for treatment were also used to adjust the position of the MVCT study relative to the kVCT study when creating the merged kVCT-MVCT images. These registration values were determined by using semiautomated registration software on the helical tomotherapy treatment console. The 6-mm slices of the MVCT studies were changed to 3-mm slices by interpolation to correspond with the slice spacing of the $\mathrm{kVCT}$ studies. The original contours used for planning on the kVCT studies were overlaid on the merged images, and they were altered by the investigators to reflect changes in target and lung volumes based on the merged kVCT-MVCT images. The only structures that typically required modification on the merged images were the lungs (to account for changes in atelectasis or pleural effusion) and the GTVs and planning target volumes (PTVs; to account for target volume changes). A three-dimensional (3D) margin of $12 \mathrm{~mm}$ (corresponding to the original margins for the initial plans) was added to the GTVs to generate the PTVs. Although 4D-CT was not performed on these patients to quantify respiratory motion, Schwarz et al. (18) recommended a 10-mm GTV-PTV margin for patients with breathing amplitudes of up to $10 \mathrm{~mm}$ undergoing intensity-modulated radiotherapy. Because GTV-PTV margins are anatomy and patient specific, a margin of $12 \mathrm{~mm}$ was chosen to ensure target coverage beyond that provided by a $10-\mathrm{mm}$ margin.

The merged images created with the MVCT scans from 3 patients were transferred to the TomoTherapy planning station to create adapted plans. Using a merged image for planning radiation delivery assumes the patient's anatomy outside the region of interest covered by the MVCT has not changed since planning and that the dose calculation using merged images is accurate.

At the London Regional Cancer Program, we have planned and treated prostate cancer patients with hip prostheses using merged images. Accuracy of dose calculation with merged image plans previously was confirmed for sites in the head and neck, lung, and prostate by creating a merged image plan and testing the delivery of the plan on tomotherapy by using three ion chamber measurements and one film exposure in a cylindrical phantom as quality assurance.

Three patients were investigated with this adaptive planning method using merged images to find out whether plan improvements can be significant for the patient and distinguish between clinically insignificant or significant anatomy changes based on their adaptive planning potential. To evaluate the quality of the adapted plans, the cumulative doses to the PTV and lungs are reported. The cumulative dose, $D_{c}$, is defined as the total dose delivered to a region of interest during the course of treatment. When using multiple adapted plans, where $i$ is the plan number $(i=1,2,3, \ldots), d_{i}$ is the planned dose to the region of interest, $n_{i}$ is the number of fractions for which plan $i$ was used, and $n_{t}$ is the prescribed number of fractions, cumulative dose:

$$
D_{c}=\frac{\sum_{i} d_{i} n_{i}}{n_{t}}
$$

Cumulative doses were calculated for the 3 patients based on the adapted plans using merged images as the planning image set. The adapted plans (APs) are referred to by the fraction number of the MVCT used to create the merged image, so $\mathrm{AP}_{10}$ is an adapted plan created using the MVCT from the tenth fraction of treatment. Doses to $99 \%$ and $1 \%$ of the PTV, along with mean lung dose (MLD) and volume of lung tissue that receives more than $20 \mathrm{~Gy}$ $\left(\mathrm{V}_{20}\right)$, were compared $(19,20)$. 
Table 1. Patient and treatment characteristics

\begin{tabular}{|c|c|c|c|c|c|c|c|c|c|c|c|}
\hline $\begin{array}{c}\text { Patient } \\
\text { no. }\end{array}$ & $\begin{array}{c}\text { Age } \\
(y) / \operatorname{Sex}\end{array}$ & Group & Histology & Stage & Plans & $\begin{array}{l}\text { Prescription } \\
\text { dose*/no. of } \\
\text { fractions }\end{array}$ & $\begin{array}{l}\text { Treatment } \\
\text { time (d) }\end{array}$ & MVCT no. & $\begin{array}{c}\text { Initial } \\
\text { GTV (ml) }\end{array}$ & $\begin{array}{c}\text { Total } \\
\text { GTV } \\
\text { change }(\%)\end{array}$ & $\begin{array}{c}\text { Mean } \\
\text { GTV } \\
\text { change/d }(\%)\end{array}$ \\
\hline 1 & $85 / \mathrm{M}$ & A & $\mathrm{LC}$ & IIIA & 2 & $60 / 30$ & 53 & 30 & 202 & -87 & -1.65 \\
\hline 2 & $68 / \mathrm{F}$ & B & A & IIIB & 2 & $60 / 30$ & 65 & 33 & 100 & -71 & -1.09 \\
\hline 3 & $67 / \mathrm{M}$ & B & $\mathrm{S}$ & IIIB & 1 & $60 / 30$ & 54 & 29 & 51 & -39 & -0.72 \\
\hline 4 & $76 / \mathrm{M}$ & B & $\mathrm{S}$ & IIIA & 1 & $60 / 30$ & 44 & 30 & 156 & -52 & -1.18 \\
\hline 5 & $60 / \mathrm{M}$ & A & A & IIIA & 2 & $60 / 30$ & 49 & 31 & 115 & -22 & -0.45 \\
\hline 6 & $76 / \mathrm{M}$ & $\mathrm{B}$ & $\mathrm{LC}$ & IIIB & 1 & $60 / 30$ & 49 & 28 & 33 & -57 & -1.16 \\
\hline 7 & $70 / \mathrm{M}$ & B & A & IIIB & 1 & $60 / 30$ & 46 & 30 & 174 & -38 & -0.82 \\
\hline 8 & $45 / \mathrm{F}$ & $\mathrm{C}$ & A & IV & 1 & $50 / 25$ & 42 & 25 & 171 & -18 & -0.73 \\
\hline 9 & $60 / \mathrm{M}$ & $\mathrm{C}$ & $\mathrm{S}$ & IV & 1 & $60 / 30$ & 44 & 29 & 3 & -41 & -0.92 \\
\hline 10 & $76 / \mathrm{M}$ & $\mathrm{B}$ & $\mathrm{S}$ & IIIB & 1 & $63 / 30$ & 47 & 31 & 41 & -42 & -0.89 \\
\hline 11 & 71/M & A & A & IIIA & 2 & $64 / 32$ & 52 & 30 & 250 & -44 & -0.86 \\
\hline 12 & $70 / \mathrm{M}$ & B & $\mathrm{S}$ & IIIA & 1 & $60 / 30$ & 44 & 30 & 132 & -32 & -0.67 \\
\hline 13 & $70 / \mathrm{M}$ & A & NSC & IIIA & 2 & $60 / 30$ & 48 & 30 & 108 & -41 & -0.80 \\
\hline 14 & 71/M & $\mathrm{C}$ & NSC & IIIA & 1 & $60 / 30$ & 51 & 29 & 38 & -15 & -0.34 \\
\hline 15 & $77 / \mathrm{M}$ & A & NSC & IIIA & 1 & $60 / 30$ & 45 & 31 & 485 & -20 & -0.43 \\
\hline 16 & $77 / F$ & B & A & IIIB & 2 & $50 / 25$ & 49 & 25 & 65 & -12 & -0.24 \\
\hline 17 & $76 / \mathrm{F}$ & $\mathrm{C}$ & $S$ & IIIA & 1 & $62 / 31$ & 49 & 31 & 36 & -23 & -0.45 \\
\hline Mean & 70 & & & & & & 48 & 30 & 127 & -38 & -0.79 \\
\hline SD & 9 & & & & & & 8 & 2 & 115 & 20 & 0.36 \\
\hline
\end{tabular}

Abbreviations: $\mathrm{LC}=$ large cell carcinoma; $\mathrm{A}=$ adenocarcinoma; $\mathrm{S}=$ squamous cell carcinoma; $\mathrm{NSC}=$ non-small cell; $\mathrm{MVCT}=$ megavoltage computed tomography; GTV = gross tumor volume.

* Dose prescribed to $95 \%$ of the planning target volume.

\section{RESULTS}

As listed in Table 1, average initial GTV for the 17 patients was $127 \pm 115$ (SD) $\mathrm{cm}^{3}$. Average total GTV change observed was $-38 \% \pm 20 \%$, ranging from a $12-87 \%$ decrease in tumor volume. Mean volume change per day was $-0.79 \% \pm 0.36 \%$, with a range of $0.24-1.65 \%$ decrease per day. There was no correlation of either rate of volume change or total volume change with initial tumor volume or of volume changes with radiotherapy treatment time. Histologic characteristics and staging did not affect rate or overall tumor volume decrease according to our data. Multiple regression analysis was carried out for such patient characteristics as PTV-lung overlap, ipsilateral-contralateral lung ratio (21), tumor density, and tumor size, but no significant correlation with tumor regression among any combination of these was found.

Three general patterns of tumor volume changes were observed in these 17 patients. Group A (5 patients) experienced an initial period of small tumor volume change, followed by a sharp decrease in tumor volume and subsequent plateau, as shown in Fig. 1. Group B (8 patients) had a more gradual linearly decreasing tumor volume, as shown in Fig. 2. Group C (4 patients) experienced variable volume changes with no clear trend toward a decrease in volume (Fig. 3).

Each of these three groups offers different opportunities for adaptive radiotherapy planning. There is a trade-off between cost, timing, and number of adaptive plans one may devise. For example, Table 2 lists cumulative doses using adapted plans at various points in the treatment of 1 patient in Group A (Patient 1; Fig. 1). Creating more adapted plans will increase resource utilization, but potentially, $\mathrm{V}_{20}$ and MLD will improve. If the adapted plan is created near the end of treatment, the overall cumulative dose will be affected very little by the improved plan because it will be used for treatment over fewer fractions. The rate and extent of GTV reduction is variable and patient dependent which determines the optimal timing and potential impact of replanning. For example, Patient 1 had a dramatically high response in a short time and thus was well positioned to benefit from replanning. The decrease in MLD and $V_{20}$ to Patient 1 as a result of adaptive planning and the time at which the plan was made may not be applicable to the other patients in Group A, who had less dramatic and more delayed GTV changes.

Table 3 lists cumulative doses using adapted plans for 2 patients (Patients 6 and 10) from Group B. Patient 6 had a higher MLD and $V_{20}$ than Patient 10 for the initial kVCT plan. The adapted plan for Patient 6 was made after a 31\% GTV decrease during 25 days and 16 fractions, and for Patient 10, after a $27 \%$ GTV decrease during 32 days and 21 fractions. For similar tumor volume changes contoured on MVCT studies, the adapted plan was capable of improving the treatment of Patient 6, who had higher lung doses compared with the treatment of Patient 10, who had lower lung doses. Group C offers little benefit for adapted plans because of the delayed tumor response, which usually results in a less than $25 \%$ volume decrease late in the treatment course. Although this is the case in this study, if anatomic changes had occurred because of atelectatic regression or change in tumor shape, adaptive planning may have been appropriate for Group $\mathrm{C}$ patients.

\section{DISCUSSION}

The GTV changes observed on helical tomotherapy by Kupelian et al. (10) and Siker et al. (11) are compared with 


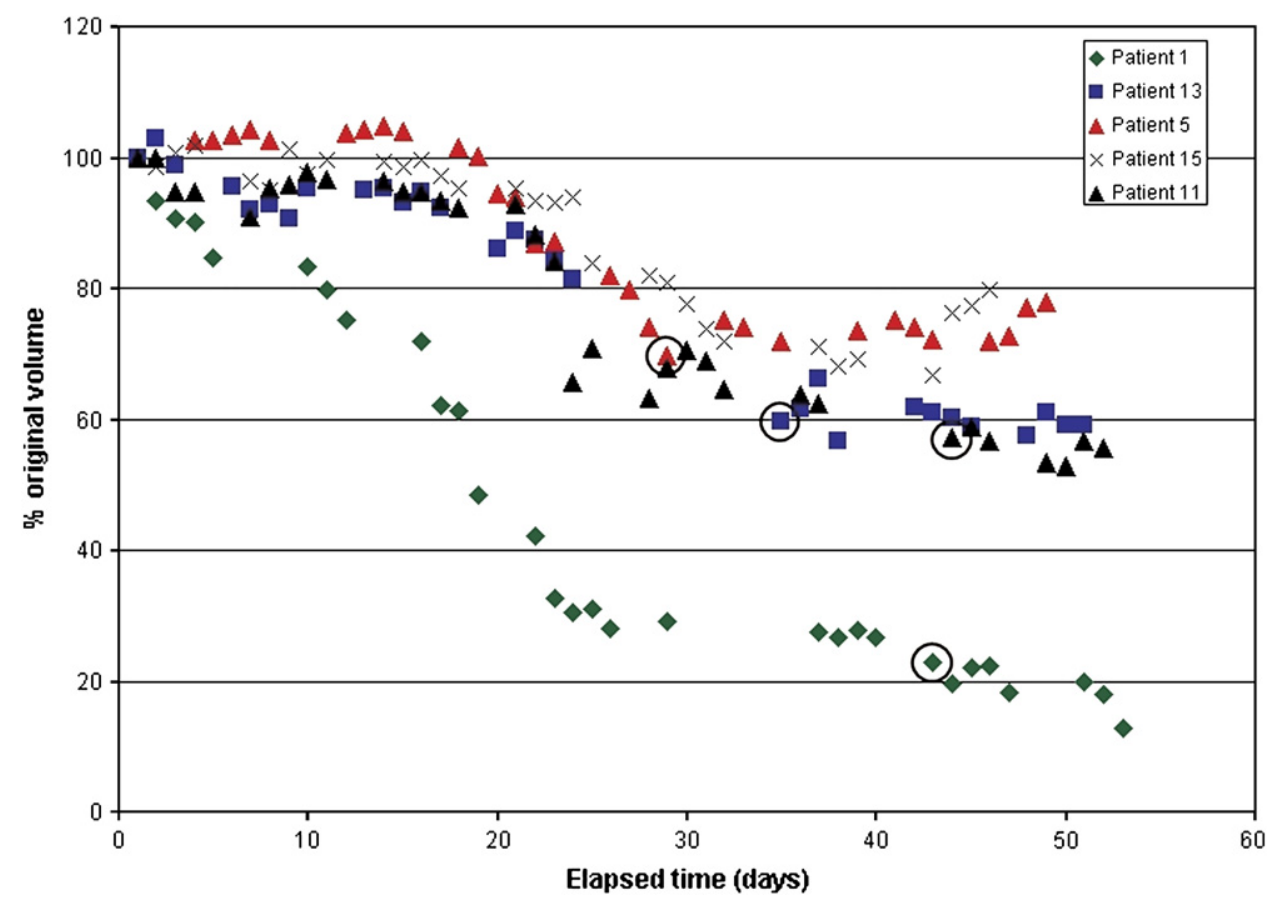

Fig. 1. Gross tumor volume changes for patients in Group A. Circles show days the adapted plans were used for Patients 1 , 5,11 , and 13 .

results of our study in Table 4, using criteria of Siker et al. (11) for complete, partial, and marginal responses or stable disease. The GTV changes noted by Kupelian et al. (10) are greater than the results reported here (22). However, our study shows greater marginal response and less stable disease than the study by Siker et al. (11). In part, this is because complete data from Siker et al. (11) include a mixture of treatment strategies: patients treated palliatively and with extracranial stereotactic radioablation. Our study included only patients treated with conventional definitive treatment (see Table 1), and when the palliative and extracranial stereotactic radioablation patient groups are excluded from the data from Siker et al. (11), there is better agreement with our study (see Siker* column in Table 4). Total doses in this study are

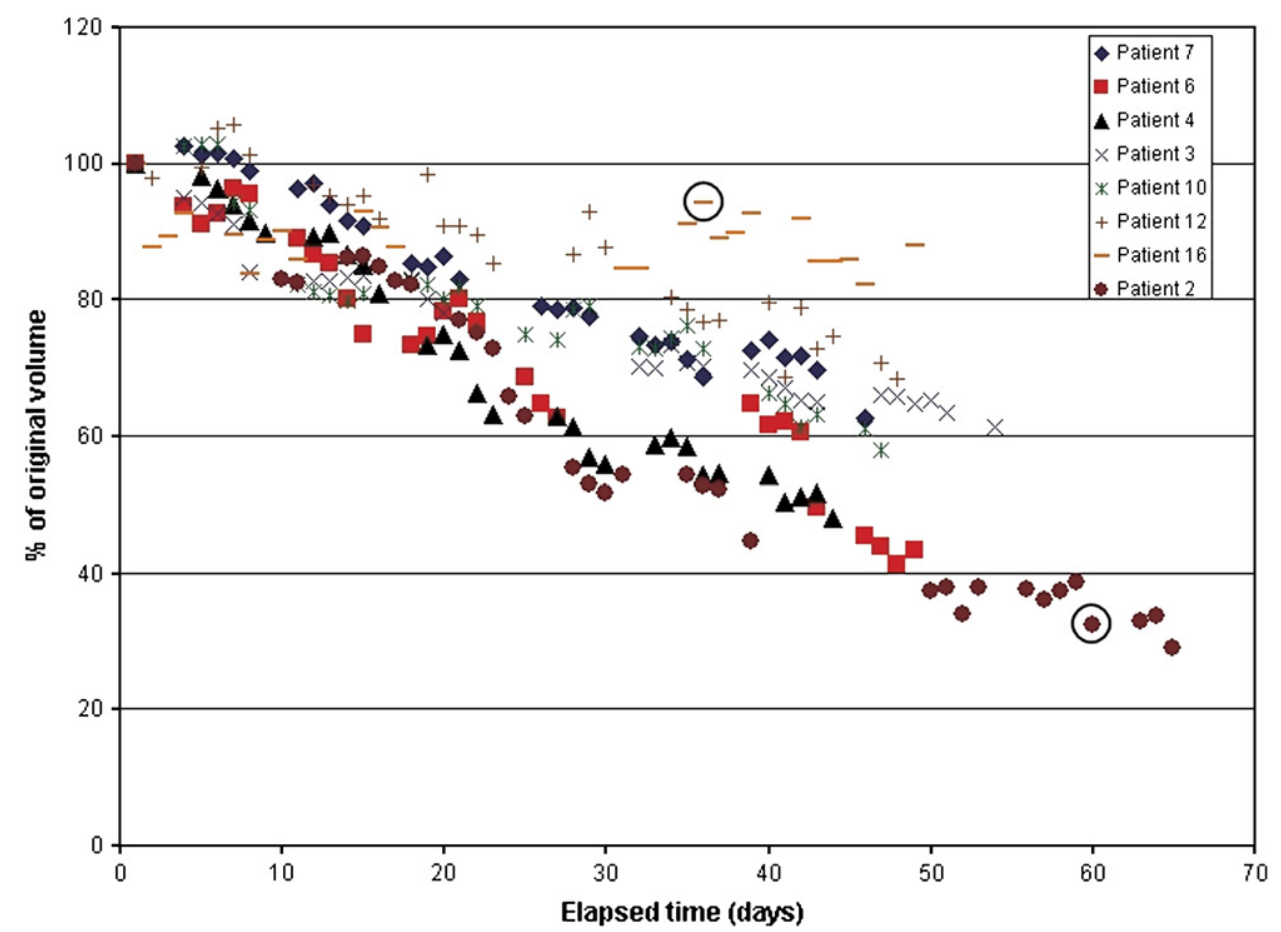

Fig. 2. Gross tumor volume changes for patients in Group B. Circles show days the adapted plans were used for Patients 2 and 16. 


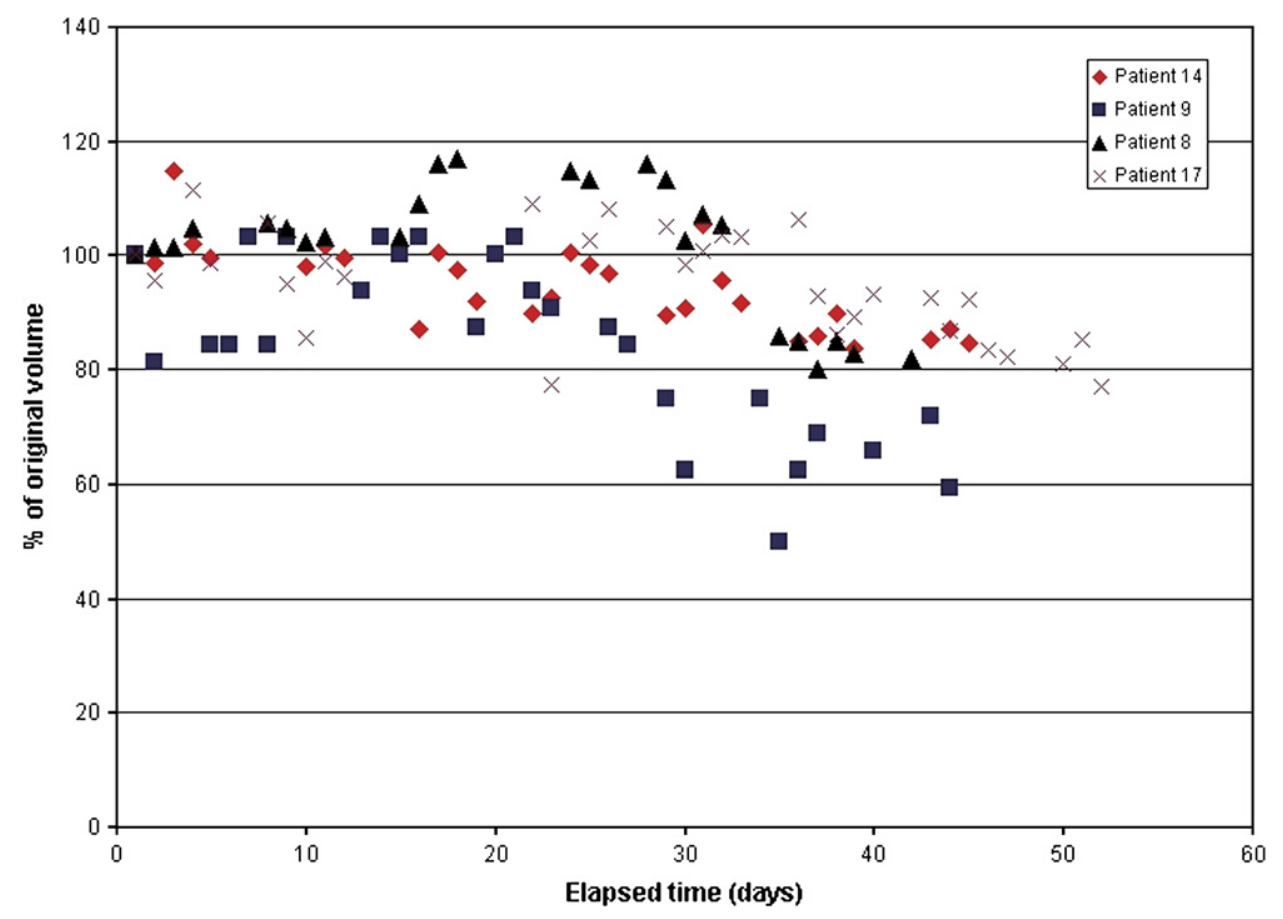

Fig. 3. Gross tumor volume changes for patients in Group C.

similar to those of Siker et al. (11), but the patients treated radically in the study by Siker et al. (11) received prescriptions based on a hypofractionation treatment that lasted 5 weeks, whereas the patients treated radically in our study were treated during a mean time of 7 weeks, ranging from 3.5-9 weeks. The higher response rates in our study may be a result of the longer treatment periods given that tumor response to radiotherapy increases with time (to a point). As noted by Siker et al. (11), their response rate observed during the course of treatment could be higher had they not used a dose-per-fraction escalation strategy that resulted in a shorter total treatment time.

Repeated adapted plans using an additional kVCT study were used for treatment of 6 patients (see Table 1). The decision to replan was made by the treating radiation oncologist based on the extent of change in the patient's internal anatomy as a result of GTV change. The primary advantage for replanning lies in greater normal tissue sparing without detrimental effects on PTV dose coverage. We showed that this aim can be met by using merged MVCT and KVCT studies with some structures previously contoured on the planning kVCT. The adapted plan data listed in Tables 2 and 3 show that adaptive planning before the 22nd fraction in the treatment is more beneficial, and inferior plans can benefit more from adaptive planning than very good plans. Also, adaptive planning can yield significant improvements in cumulative doses to organs at risk (OARs) after a GTV decrease of approximately $30 \%$ or more, provided that decrease occurs at approximately the first 15-20 fractions of treatment, which happened in $40 \%$ of the patients in the study. The pattern of regression is a main end point in this study because it determines the optimal point for adaptive planning. If the patient belongs to Group A, an adapted plan may be beneficial at the end of the high rate tumor regression to improve the therapeutic ratio during treatment in the plateau region. Patients in Group B should be considered for adapted plans only if their tumor volume decreases by more than $30 \%$ in the first 22 fractions, whereas adaptive planning benefits for patients in Group C will not be significant in most cases.

The $30 \%$ threshold mentioned is not intended to be a concrete principle, but is useful as a criterion to identify or "flag" patients for adaptive planning evaluation. GTV is an informative parameter in this respect and was chosen as a criterion

Table 2. Cumulative doses for adapted plan combinations for Patient 1 from Group A

\begin{tabular}{|c|c|c|c|c|c|c|c|c|}
\hline & No AP & $\mathrm{AP}_{10}$ & $\mathrm{AP}_{15}$ & $\mathrm{AP}_{10}+\mathrm{AP}_{15}$ & $\mathrm{AP}_{22}$ & $\mathrm{AP}_{10}+\mathrm{AP}_{22}$ & $\mathrm{AP}_{15}+\mathrm{AP}_{22}$ & $\mathrm{AP}_{10}+\mathrm{AP}_{15}+\mathrm{AP}_{22}$ \\
\hline PTV D 1 (Gy) & 62.6 & 62.2 & 63.1 & 63.0 & 62.3 & 62.0 & 62.5 & 62.4 \\
\hline PTV D 99 (Gy) & 58.4 & 58.3 & 57.8 & 57.7 & 58.6 & 58.5 & 58.3 & 58.2 \\
\hline $\mathrm{V}_{20}(\mathrm{~Gy})$ & 27.7 & 26.6 & 24.9 & 24.6 & 25.6 & 25.0 & 24.3 & 24.0 \\
\hline
\end{tabular}

Abbreviations: $\mathrm{AP}_{\mathrm{x}}=$ adapted plan after $\mathrm{x}$ fractions; $\mathrm{AP}_{\mathrm{x}}+\mathrm{AP}_{\mathrm{y}}=$ adapted plan after $\mathrm{x}$ fractions and after $\mathrm{y}$ fractions; $\mathrm{D}_{\mathrm{x}}=\mathrm{dose}$ planned to $\mathrm{x} \%$ of the PTV; MLD = mean lung dose; PTV = planning target volume; $\mathrm{V}_{20}=$ volume of lung receiving greater than $20 \mathrm{~Gy}$. 
Table 3. Cumulative doses for adapted plan combinations for Patients 6 and 10 from Group B

\begin{tabular}{|c|c|c|c|c|c|c|}
\hline & \multicolumn{3}{|c|}{ Patient 6} & \multicolumn{3}{|c|}{ Patient 10} \\
\hline & $\begin{array}{l}\text { No } \\
\text { AP }\end{array}$ & $\mathrm{AP}_{16}$ & $\begin{array}{l}\text { Change in } \\
\text { cumulative } \\
\text { dose }(\%)\end{array}$ & $\begin{array}{l}\text { No } \\
\text { AP }\end{array}$ & $\mathrm{AP}_{21}$ & $\begin{array}{c}\text { Change in } \\
\text { cumulative } \\
\text { dose }(\%)\end{array}$ \\
\hline PTV D $_{1}(\mathrm{~Gy})$ & 61.9 & 62.3 & 0.6 & 65.8 & 65.7 & -0.2 \\
\hline PTV D 99 (Gy) & 59.4 & 59.2 & -0.4 & 61.9 & 62.0 & 0.1 \\
\hline MLD (Gy) & 15.1 & 14.5 & -4.1 & 10.3 & 10.0 & -2.4 \\
\hline $\mathrm{V}_{20}(\mathrm{~Gy})$ & 26.1 & 24.2 & -7.4 & 15.1 & 14.7 & -3.2 \\
\hline
\end{tabular}

Abbreviations: $\mathrm{AP}_{\mathrm{x}}=$ adapted plan after $\mathrm{x}$ fractions; $\mathrm{D}_{\mathrm{x}}=$ dose planned to $\mathrm{x} \%$ of the PTV; MLD = mean lung dose; PTV = planning target volume; $\mathrm{V}_{20}=$ volume of lung receiving greater than $20 \mathrm{~Gy}$.

because it is easy to evaluate radiologically. The possibility of benefiting from adaptive planning is suggested when a patient reaches or crosses this threshold, but actual benefits also rely on such variables as the existence of elective nodal irradiation or proximity of organs at risk. In cases in which organs at risk are dose limiting, it would be apt to evaluate the adaptive planning potential sooner and more often. When a patient's GTV does not cross the suggested threshold and is not accompanied by significant anatomy changes, it is not necessary to use resources creating and evaluating the need for adapted plans. Implementation of such a strategy does not rely on the availability of onboard CT imaging. A repeated CT simulation during the third or fourth week of treatment could accomplish the goal of identifying patients who could benefit from replanning.

Haasbeek et al. (16) showed that adaptive planning for patients with Stage I NSCLC undergoing stereotactic radiotherapy was not necessary based on repeated 4D CTs after 2-12 days after the first stereotactic fraction. Our study covers a longer period and the patients have more advanced disease, which is likely why our conclusions about adaptive planning differ. Siebert et al. (23) were successful in their efforts to predict GTV change in a group of 19 patients treated with helical tomotherapy. Their empirical model was based on a locally weighted regression learning algorithm. In our patient set, we could not measure any tumor or patient characteristics or combination thereof that correlated with GTV change.

Deformable registration was not used in this study to calculate cumulative doses to OARs and target volumes, but

Table 4. Comparison of data with previous studies

\begin{tabular}{lccccc}
\hline & & \multicolumn{4}{c}{ Frequency $(\%)$} \\
\cline { 3 - 6 } Response & $\begin{array}{c}\text { Reduction in } \\
\text { volume }(\%)\end{array}$ & LRCP & Kupelian & Siker & Siker* \\
\hline Complete & 100 & 0 & 0 & 0 & 0 \\
Partial & $65-99$ & 12 & 80 & 12 & 18 \\
Marginal & $35-64$ & 47 & 20 & 20 & 29 \\
Stable & $0-34$ & 41 & 0 & 68 & 53 \\
$\quad$ disease & & & & & \\
\hline
\end{tabular}

Abbreviation: $\mathrm{LRCP}=$ London Regional Cancer Program.

* There are two different data sets from Siker. could be a valuable extension. Were this technique used, more specific definition of doses to all structures could be determined with reliable dose-volume histograms. The biggest advantage it could endow is the ability to create adapted plans that compensate for underdosage or overdosage of targets or OARs. In addition to GTV change, more criteria for adapted planning potential could involve patients who experienced a variation from the planned dose greater than a threshold value.

Although GTV changes are caused mostly by cancer reduction, the observations reported here may be affected by other factors, such as breathing or atelectatic changes. Breathing patterns can change during the course of treatment (24). The apparent volume visible on the MVCT will change if breathing amplitude changes because MVCT acquisition is similar to a slow CT scan. The GTV motion creates artifacts that increase the contouring uncertainty and result in an image that is not exactly reflective of the actual tumor position or size (25).

The GTVs alone may not completely reveal the response to radiotherapy because neither $\mathrm{kVCT}$ nor MVCT imaging techniques can reliably detect tumor composition. Although tumors may not decrease much in volume, necrosis, nonmalignant tissue, and inflammation could replace malignant tissue, but may be indistinguishable by using CT (26). It also was shown that tumor composition was heterogeneous and tumor volumes were not reflective of the amount of actively replicating cancerous tissue (27). This result could explain why Kupelian et al. (10) observed a more rapid decrease in GTV for larger tumors, but unfortunately that result was not reproduced in the present study or by Siker et al (11). It also is not clear yet whether regressing tumors leave behind nests of cells that should be treated further or whether smaller fields still adequately encompass subclinical disease. Our hypothesis is that if the gross tumor regresses, the microscopic extension disappears proportionally, and reducing the PTV would not result in decreased coverage of diseased tissue, but this conjecture is unconfirmed to date. Microscopic extension of NSCLC was quantified in postoperative specimens, but studying its change during the course of radiotherapy is impossible by using histologic techniques because the tumor is surgically excised (28). Ideally, serial biologicbased imaging acquired during a course of radiation would be available to provide information in addition to the tissue density change information available with $\mathrm{CT}$ studies. Unfortunately, this adds to the logistic complexity of the replanning process, even when using such existing biologic imaging as positron emission tomography, but it is still an area worth exploring.

\section{CONCLUSION}

We observed decreases in GTV in 17 patients with NSCLC during the standard course of fractionated treatment with helical tomotherapy. Significant improvement in cumulative doses by using adapted plans can be made after tumor volume decreases by greater than $30 \%$ within the first 20 treatment fractions. In $40 \%$ of patients in this study, GTV 
changes were of sufficient magnitude and occurred sufficiently early in the treatment course that one could realistically anticipate that adapted radiotherapy would improve the therapeutic ratio and clinical results. Continuation of this study on a larger patient base will lead to the formulation of guidelines regarding adaptive planning based on merged image plans and to improved ability to identify patients before treatment who may require later adapted planning.

\section{REFERENCES}

1. Forrest LJ, MacKie TR, Ruchala K, et al. The utility of megavoltage computed tomography images from a helical tomotherapy system for setup verification purposes. Int J Radiat Oncol Biol Phys 2004;60:1639-1644.

2. Langen KM, Zhang Y, Andrews R, et al. Initial experience with megavoltage (MV) CT guidance for daily prostate alignments. Int J Radiat Oncol Biol Phys 2005;62:1517-1524.

3. Meeks SL, Harmon JF, Langen KM, et al. Performance characterization of megavoltage computed tomography imaging on a helical tomotherapy unit. Med Phys 2005;23:2673-2681.

4. Song WY, Chiu B, Bauman GS, et al. Prostate contouring uncertainty in megavoltage computed tomography images acquired with a helical tomotherapy unit during image-guided radiation therapy. Int J Radiat Oncol Biol Phys 2006;65: 595-607.

5. Welsh JS, Bradley K, Ruchala KJ, et al. Megavoltage computed tomography imaging: A potential tool to guide and improve the delivery of thoracic radiation therapy. Clin Lung Cancer 2004; 5:303-306.

6. Yartsev S, Dar AR, Woodford C, et al. Initial experience in treating lung cancer with helical tomotherapy. Biomed Imaging Interv J 2007;3:e2. Accessable at: http://www.biij.org/2007/1/ e2.

7. Dubben HH, Thames HD, Beck-Bornholdt HP. Tumor volume: A basic and specific response predictor in radiotherapy. Radiother Oncol 1998;47:167-174.

8. Willner J, Baier K, Caragiani E, et al. Dose, volume, and tumor control predictions in primary radiotherapy of non-small-cell lung cancer. Int J Radiat Oncol Biol Phys 2002;52:382-389.

9. Werner-Wasik M, Xiao Y, Pequignot E, et al. Assessment of lung cancer response after nonoperative therapy: Tumor diameter, bidimensional product, and volume. A serial CT scan-based study. Int J Radiat Oncol Biol Phys 2001;51:56-61.

10. Kupelian PA, Ramsey C, Meeks SL, et al. Serial megavoltage CT imaging during external beam radiotherapy for non-smallcell lung cancer: Observations on tumor regression during treatment. Int J Radiat Oncol Biol Phys 2005;63:1024-1028.

11. Siker ML, Tomé WA, Mehta MP. Tumor volume changes on serial imaging with megavoltage CT for non-small-cell lung cancer during intensity-modulated radiotherapy: How reliable, consistent, and meaningful is the effect? Int $J$ Radiat Oncol Biol Phys 2006;66:135-141.

12. Ramsey CR, Langen KM, Kupelian PA, et al. A technique for adaptive image-guided helical tomotherapy for lung cancer. Int J Radiat Oncol Biol Phys 2006;64:1237-1244.

13. Lagerwaard FJ, Underberg RWM, Slotman BJ, et al. Time trends in target volumes during stereotactic radiotherapy for stage I non-small cell lung cancer (NSCLC). Radiother Oncol 2006;73(Suppl.):S27-S28.

14. Underberg RWM, Lagerwaard FJ, van Tinteren $\mathrm{H}$, et al. Time trends in target volumes for stage I non-small-cell lung cancer after stereotactic radiotherapy. Int J Radiat Oncol Biol Phys 2006;64:1221-1228.
15. Bosmans G, van Baardwijk A, Dekker A, et al. Intra-patient variability of tumor volume and tumor motion during conventionally fractionated radiotherapy for locally advanced nonsmall-cell lung cancer: A prospective clinical study. Int J Radiat Oncol Biol Phys 2006;66:748-753.

16. Haasbeek CJA, Lagerwaard FJ, Cuijpers JP, et al. Is adaptive treatment planning required for stereotactic radiotherapy of stage I non-small-cell lung cancer? Int J Radiat Oncol Biol Phys 2007;67:1370-1374.

17. Erridge SC, Seppenwoolde Y, Muller SH, et al. Portal imaging to assess set-up errors, tumor motion and tumor shrinkage during conformal radiotherapy of non-small cell lung cancer. Radiother Oncol 2003;66:75-85.

18. Schwarz M, Van Der Geer J, Van Herk M, et al. Impact of geometrical uncertainties on 3D-CRT and IMRT dose distributions for lung cancer treatment. Int J Radiat Oncol Biol Phys 2006;65: $1260-1269$.

19. Graham MV, Purdy JA, Emami B, et al. Clinical dose-volume histogram analysis for pneumonitis after 3D treatment for nonsmall-cell lung cancer (NSCLC). Int J Radiat Oncol Biol Phys 1999;45:323-329.

20. Rodrigues G, Lock M, D'Souza D, et al. Prediction of radiation pneumonitis by dose-volume histogram parameters in lung cancer-A systematic review. Radiother Oncol 2004;71:127-138.

21. Yartsev S, Chen J, Yu E, et al. Comparative planning evaluation of intensity-modulated radiotherapy techniques for complex lung cancer cases. Radiother Oncol 2006;78:169-176.

22. Dar AR, Yartsev S, Rodrigues G, et al. Serial megavoltage CT imaging during external beam radiotherapy for non-small-celllung cancer: In regard to Kupelian, et al. Int J Radiat Oncol Biol Phys 2005;630:1024-1028.

23. Seibert RM, Ramsey CR, Hines JW, et al. A model for predicting lung cancer response to therapy. Int J Radiat Oncol Biol Phys 2007;67:601-609.

24. Britton KR, Starkschall G, Tucker SL, et al. Assessment of gross tumor volume regression and motion changes during radiotherapy for non-small-cell lung cancer as measured by four-dimensional computed tomography. Int J Radiat Oncol Biol Phys 2007;68:1036-1046.

25. Smeenk C, Gaede S, Battista JJ. Delineation of moving targets with slow MVCT scans: Implications for adaptive non-gated lung tomotherapy. Phys Med Biol 2007;52:1119-1134.

26. MacVicar D, Husband JE. Assessment of response following treatment for malignant disease. BJR Suppl 1997;70:S41-S49.

27. Bentzen SM, Thames HD. Tumor volume and local control probability: Clinical data and radiobiological interpretations. Int J Radiat Oncol Biol Phys 1996;36:247-251.

28. Giraud P, Antoine M, Larrouy A, et al. Evaluation of microscopic tumor extension in non-small cell lung cancer for three-dimensional conformal radiotherapy planning. Int $J$ Radiat Oncol Biol Phys 2000;48:1015-1024. 